\title{
Protective effects of catalpol on mitochondria of hepatocytes in cholestatic liver injury
}

\author{
XINGJUAN GAO, JIAJU XU and HONGBO LIU \\ Department of Pediatrics, The Affiliated Yantai Yuhuangding Hospital of Qingdao University, \\ Yantai, Shandong 264000, P.R. China
}

Received January 5, 2020; Accepted May 21, 2020

DOI: $10.3892 / \mathrm{mmr} .2020 .11337$

\begin{abstract}
Cholestasis, which is caused by the obstruction of bile flow, can lead to rapid organ injury, cell apoptosis and necrosis of hepatocytes, and may eventually develop into fibrosis and cirrhosis. Oxidative stress and mitochondrial dysfunction are the key pathogenic signs of hepatic cholestasis. Catalpol has pharmacological activities, including antioxidative and anti-inflammatory effects, and may relieve mitochondrial damage and restore mitochondrial membrane potential. However, the potential roles and mechanisms of catalpol in cholestasis-induced liver injury are not clear. In the present study, liver function-related indexes were measured in the serum of mice by commercial kits. In addition, levels of serum inflammatory factors were detected by ELISA. Hematoxylin and eosin staining was performed to observe histopathological changes, and mitochondrial membrane potential was detected using JC-1 staining. Mitochondrial adenosine triphosphate (ATP), reactive oxygen species (ROS) and malondialdehyde levels were determined using a luciferase reporter kit, flow cytometry and a thiobarbituric acid reactive substance assay kit, respectively. Western blotting was performed to detect the expression levels of apoptosis-related proteins in liver tissues. The findings revealed that catalpol reduced liver damage caused by cholestasis, improved the mitochondrial membrane potential, and increased the ATP content and glutathione content of cholestasis model mice. Moreover, catalpol also reduced the ROS level, inhibited lipid
\end{abstract}

Correspondence to: Dr Hongbo Liu, Department of Pediatrics, The Affiliated Yantai Yuhuangding Hospital of Qingdao University, 20 East Yuhuangding Road, Zhifu, Yantai, Shandong 264000, P.R. China

E-mail: liuhongb_hbo@163.com

Abbreviations: H\&E, hematoxylin and eosin; ATP, adenosine triphosphate; ROS, reactive oxygen species; GSH, glutathione; MDA, malondialdehyde; BDL, bile duct ligation; ALT, alanine aminotransferase; AST, aspartate aminotransferase; ALP, alkaline phosphatase; TBIL, total bilirubin; TBA, total bile acid

Key words: catalpol, mitochondria, cholestasis, oxidative stress peroxidation, and regulated oxidative stress and apoptotic protein expression. Thus, the present study preliminarily confirmed that catalpol can reduce liver injury in a mouse model of cholestasis through inhibiting oxidative stress and enhancing mitochondrial membrane potential.

\section{Introduction}

Cholestasis, which is characterized by accumulation of bile acids in the liver or systemic circulation, leads to bile retention and can develop into symptoms such as fibrosis and cirrhosis, accompanied by inflammation $(1,2)$. Cholestasis is mainly caused by mechanical obstruction or a genetic defect at the bile duct $(3,4)$. A previous study reported that protein deficiency or a dysfunction caused by genetic defects may have an important role in the formation, progression and excretion of bile (3).

Oxidative stress and mitochondrial dysfunction are regarded as the main cellular symptoms of hepatic cholestasis (5). Excessive production of reactive oxygen species (ROS) can mediate mitochondrial oxidative damage, such as destruction of the respiratory chain, along with destruction of the inner and outer membrane, and various matrix proteins (6-8). Furthermore, changes in mitochondrial membrane permeability may result in the increased expression of various pro-apoptotic factors, such as cytochrome $c$, thus initiating the mitochondrial apoptosis pathway to induce cell apoptosis (9). Catalpol, an iridoid glycoside abundant in the roots of Rehmannia glutinosa, has been reported to protect the body from developing neurodegenerative diseases; it has been shown to improve the cognitive function of rats in old age (10), reduce oxidative stress in the cerebral cortex and ameliorate the pathological condition of Alzheimer's disease in mice (11). In addition, catalpol has a variety of pharmacological activities, including anti-inflammatory effects, and has been reported to play an important role in maintaining a redox balance (12). A recent study revealed that catalpol may improve neointimal hyperplasia in hyperglycemic rats through reducing the expression of monocyte chemoattractant protein-1 in the carotid artery, thus suggesting that catalpol could have a role in the prevention of hyperglycemia (13). Another study reported that catalpol exerted a protective effect on lipopolysaccharide/D-galactosamine-induced acute liver injury through the inhibition of inflammation and oxidation, and regulation of related signaling pathways (14). 
Furthermore, it has been demonstrated that catalpol can alleviate mitochondrial damage (15). In addition, catalpol has been demonstrated to inhibit the production of inflammatory mediators in human intestinal Caco-2 cells, pointing to the potential role of catalpol in the prevention of intestinal inflammation (16).

In view of the antioxidative stress and anti-inflammatory effects of catalpol, and its ability to improve mitochondrial membrane potential, it was hypothesized that catalpol could also improve hepatic injury in cholestasis. Therefore, the present study aimed to further explore the potential mechanism of action of catalpol.

\section{Materials and methods}

Animals. BALB/C male mice ( $\mathrm{n}=30$, weight, $10-25 \mathrm{~g}$; age, 6-8 weeks) were purchased from Shanghai SLAC Laboratory Animal Co., Ltd. Mice were housed under a 12-h light/dark cycle at a relative temperature of $23 \pm 2{ }^{\circ} \mathrm{C}$ and $70 \%$ humidity. The mice were provided with free access to food and water. The animal experiments were performed strictly in accordance with the Care and Use of Laboratory Animal Regulations (17). This study was approved by the Ethics Review Board for Animal Studies of the Affiliated Yantai Yuhuangding Hospital of Qingdao University (Yantai, China; approval no. SH20186242).

Model establishment and grouping. A mouse model of cholestasis was established by bile duct ligation (BDL). Briefly, the mice were anesthetized by intraperitoneal injection of ketamine (100 mg/kg; Jiangsu Hengrui Medicine Co., Ltd.,) and xylazine (10 mg/kg; Sigma-Aldrich; Merck KGaA). Next, the common bile duct was separated from its surrounding connective tissue, and the hepatic artery from the portal vein. The common bile duct was then ligated with 5-0 silk thread at two locations close to the beginning of its intrapancreatic portion and was transected between the two ligatures. Mice in the sham group received a laparotomy without subsequent identification and ligation of the bile duct. Mice in the sham and BDL groups were administered $0.9 \%$ normal saline (20 $\mathrm{ml} / \mathrm{kg} / \mathrm{day})$ for 7 days. Mice in the catalpol-high $(-\mathrm{H})$ group were gavaged with $10 \mathrm{mg} / \mathrm{kg}$ catalpol (Nanjing Jingzhu Biotechnology Co., Ltd.) for 7 days after undergoing sham surgery, whereas those in the BDL + catalpol groups were gavaged with either $5 \mathrm{mg} / \mathrm{kg}$ catalpol [catalpol-low (-L)] or $10 \mathrm{mg} / \mathrm{kg}$ catalpol (catalpol-H) for 7 days after the BDL model was established. A total of 1 week after the experiment, all mice were sacrificed with an overdose of pentobarbital sodium $(120 \mathrm{mg} / \mathrm{kg})$ followed by collection of blood and liver samples. Blood was collected via the tail vein, and then the blood samples $(0.4 \mathrm{ml})$ were immediately separated by centrifugation at $2,200 \mathrm{x} \mathrm{g}$ at $4^{\circ} \mathrm{C}$ for $10 \mathrm{~min}$ to obtain serum samples.

Analysis of liver function-related indexes in serum. The serum of mice was collected from blood samples by centrifugation at $2,200 \mathrm{x} \mathrm{g}$ at $4^{\circ} \mathrm{C}$ for $10 \mathrm{~min}$. Commercial ELISA kits were used to measure serum levels of alanine aminotransferase (ALT; cat. no. C009-2), aspartate aminotransferase (AST; cat. no. C010-2), alkaline phosphatase (ALP; cat. no. A059-2), total bilirubin (TBIL; cat. no. C019-1) and total bile acid (TBA; cat. no. E003-2) (all Nanjing Jiancheng Bioengineering Institute), according to the manufacturer's protocol.

Determination of inflammatory factor index in serum. The blood samples were centrifuged at 2,200 x g for $10 \mathrm{~min}$ at $4^{\circ} \mathrm{C}$ to collect serum, which was then immediately stored at $-80^{\circ} \mathrm{C}$ until use. Tumor necrosis factor- $\alpha$ (TNF- $\alpha$; cat. no. MTA00B), interleukin (IL)-1 $\beta$ (cat. no. MLB00C) and IL-6 (cat. no. M6000B) were detected in the serum samples using ELISA kits (R\&D Systems, Inc.) according to the manufacturer's protocol.

Histomorphology. The liver tissues were sliced and fixed in $4 \%$ paraformaldehyde overnight at $4^{\circ} \mathrm{C}$. Subsequently, tissue sections were dehydrated in a diminishing series of ethanol, embedded in paraffin and sectioned into $4-5-\mu \mathrm{m}$ slices. The sections were stained with hematoxylin and eosin (H\&E) for $10 \mathrm{~min}$ at room temperature and observed under a light microscope (magnification, x100; DM4000B; Leica Microsystems, Inc.).

Liver mitochondria isolation. After resection of the liver, the tissue samples were homogenized in a buffer containing $2 \mathrm{mmol} / 1 \mathrm{HEPES}, 0.5 \mathrm{mmol} / 1$ EGTA, $70 \mathrm{mmol} / 1 \mathrm{mannitol}$, $220 \mathrm{mmol} / \mathrm{l}$ sucrose and 0.1\% BSA (Sigma-Aldrich; Merck $\mathrm{KGaA})$ at a ratio of 10:1 buffer:tissue (v/w), final $\mathrm{pH} 7.4$. The tissue homogenates were centrifuged at $4^{\circ} \mathrm{C}$ for $10 \mathrm{~min}$ at $1,000 \mathrm{x}$ g to pellet the whole cells and nuclei. The supernatant was further centrifuged at $4^{\circ} \mathrm{C}$ for $10 \mathrm{~min}$ at $10,000 \mathrm{x} \mathrm{g}$ to precipitate the heavy membrane fractions (mitochondria).

Mitochondrial membrane potential detection. JC-1 staining was performed to detect the mitochondrial membrane potential. The hepatocytes were isolated from the liver tissues. Briefly, the liver tissues were digested in $0.1 \%$ trypsin for $10 \mathrm{~min}$ at $37^{\circ} \mathrm{C}$ and filtered $(40 \mu \mathrm{m}$; Hangzhou Xiaoyou Biotechnology Co., Ltd.) to obtain cell suspension, and the cells were cultured in DMEM (Gibco; Thermo Fisher Scientific, Inc.) supplemented with $10 \%$ fetal bovine serum, $100 \mathrm{U} / \mathrm{ml}$ penicillin and $100 \mu \mathrm{g} / \mathrm{ml}$ streptomycin (Thermo Fisher Scientific, Inc.) at $37^{\circ} \mathrm{C}$ with $5 \% \mathrm{CO}_{2}$. The harvested hepatocytes were subsequently washed with PBS, and then incubated with $5 \mu \mathrm{mol} / 1 \mathrm{JC}-1$ (Cell Signaling Technology, Inc.) for $30 \mathrm{~min}$ prior to being subjected to flow cytometry (ACEA Bioscience; Agilent Technologies, Inc.).

Mitochondrial adenosine triphosphate (ATP) content. The mitochondria suspension and $1 \mathrm{X}$ Reaction buffer solution were prepared and subjected to a luciferase luciferin kit (cat. no. FF2021; Enliten ${ }^{\circledR}$; Promega Corporation) to determine mitochondrial ATP content, according to the manufacturer's protocol. Subsequently, luminous intensity of the sample was detected at $\lambda=560 \mathrm{~nm}$ using the FLUOstar Omega ${ }^{\circledR}$ multi-function microplate reader (BMG Labtech $\mathrm{GmbH}$ ).

Detection of ROS production. Production of intracellular ROS was examined using the fluorescent probe DCFH-DA (cat. no. HY-D0940; MedChemExpress). Liver mitochondria were isolated as aforementioned and incubated with a final 
concentration of $10 \mu \mathrm{mol} / 1 \mathrm{DCFH}-\mathrm{DA}$ for $30 \mathrm{~min}$ at $37^{\circ} \mathrm{C}$ and the fluorescence intensity was measured by flow cytometry (ACEA Bioscience; Agilent Technologies, Inc.) ( $\lambda$ excitation, $488 \mathrm{~nm} ; \lambda$ emission, $525 \mathrm{~nm}$ ).

Glutathione (GSH) content detection. The level of GSH in mitochondria and serum was determined by spectrophotometry. The chemical 5,50-dithio-2-nitrobenzoic acid (DTNB; cat. no. JD499-5G; Beijing Dingguo Changsheng Biotechnology Co., Ltd.) was used as the indicator of GSH. To extract mitochondrial GSH, the mitochondrial suspension was treated with trichloroacetic acid to a final concentration of $10 \%(\mathrm{w} / \mathrm{v})$ at room temperature for $5 \mathrm{~min}$. The denatured proteins were removed by centrifugation at $15,000 \mathrm{xg}$ for $1 \mathrm{~min}$ at $4^{\circ} \mathrm{C}$ and $100 \mu \mathrm{l} \mathrm{DTNB}$ ( $0.04 \%$ phosphate buffer) was then added at room temperature for $15 \mathrm{~min}$. The yellow intensity was recorded at $412 \mathrm{~nm}$ using an ultraviolet spectrophotometer (ultrospec $2000^{\circledR}$ ultraviolet spectrophotometer; Pharmacia Biotechnology; GE Healthcare).

Malondialdehyde (MDA) content detection. After isolation of liver mitochondria, MDA in mitochondria and serum was detected using a thiobarbituric acid reactive substance (TBARS) assay kit (cat. no. KA4409; AmyJet Scientific, Inc.) according to the manufacturer's protocol. Isolated mitochondria were washed in an ice-cold 3-(N-norpholino) propanesulfonic acid (MOPS)-KCl buffer (50 mM MOPS, $100 \mathrm{mM} \mathrm{KCl}$ ) to remove sucrose, and re-suspended in fresh MOPS- $\mathrm{KCl}$ buffer. Then, the mitochondrial suspension was mixed with $15 \%$ trichloroacetic acid, $0.375 \%$ thiobarbituric acid, $0.24 \mathrm{M} \mathrm{HCl}$, and $0.5 \mathrm{mM}$ Trolox. The mixture was heated for $15 \mathrm{~min}$ at $100^{\circ} \mathrm{C}$. Following centrifugation $(15,000 \mathrm{x} \mathrm{g}$; $5 \mathrm{~min} ; 4^{\circ} \mathrm{C}$ ), the absorbance of the supernatant was assessed, MDA in reaction with thiobarbituric acid produces the MDA-TBA adduct, which could be read at $532 \mathrm{~nm}$ using an Epoch plate reader (BioTek Instruments, Inc.).

Western blot analysis. Liver tissues $(0.1 \mathrm{~g})$ were homogenized by incubation with $1 \mathrm{ml}$ protein lysis buffer (Beyotime Institute of Biotechnology) and centrifuged at $12,000 \mathrm{x} \mathrm{g}$ for $10 \mathrm{~min}$ at $4^{\circ} \mathrm{C}$ to collect the supernatant. The concentration of the protein was evaluated using a BCA protein assay kit (Beyotime Institute of Biotechnology). Proteins $(30 \mu \mathrm{g})$ were separated by $10 \%$ SDS-PAGE and transferred onto PVDF membranes (Sigma-Aldrich; Merck KGaA). The membranes were then blocked with $5 \%$ skimmed milk powder for $3 \mathrm{~h}$ at room temperature and incubated with the relevant primary antibodies [cytochrome $c(1: 1,000$; cat. no. ab90529; 15 kD), Bcl-2 (1:1,000; cat. no. ab59348; $26 \mathrm{kD})$, Bax (1:1,000; cat. no. ab32503; $21 \mathrm{kD})$, cleaved caspase-3 (1:1,000; cat. no. ab2302; $17 \mathrm{kD})$ and caspase-3 (1:5,000; cat. no. ab32351; $32 \mathrm{kD}$ ) (all from Abcam)] overnight at $4^{\circ} \mathrm{C}$. The membranes were subsequently incubated with horseradish peroxidase-conjugated goat anti-rabbit IgG $\mathrm{H} \& \mathrm{~L}$ (1:2,000; cat. no. ab205718; Abcam) secondary antibodies for 1 h. GAPDH (1:1,000; cat. no. ab181602; 36 kD; Abcam) served as the internal control. Chemiluminescence detection was conducted using an ECL Western Blotting Substrate kit (cat. no. ab65623; Abcam). Protein expression levels were semi-quantified using the Image-Pro Plus 6.0 software (Media Cybernetics, Inc.).
Statistical analysis. The experiments were performed in triplicate. Data are presented as the mean \pm standard deviation. The data were analyzed using one-way ANOVA followed by Bonferroni's post-hoc test using SPSS 20.0 software (IBM Corporation). $\mathrm{P}<0.05$ was considered to indicate a statistically significant difference.

\section{Results}

Effects of catalpol on serum biochemical indexes of cholestatic mice. Mice in the catalpol-H treatment group showed no significant alterations in the serum levels of ALT, AST, ALP, TBIL or TBA (Fig. 1A-E) compared with mice in the sham control group. However, addition of catalpol-H and catalpol-L could significantly reduce serum ALT, AST, ALP, TBIL and TBA levels in the BDL model group (Fig. 1A-E), with catalpol-H producing a significantly greater protective effect compared with catalpol-L.

Effects of catalpol on serum inflammatory factor indexes of cholestatic mice. As shown in Fig. 1F-H, it was revealed that mice treated with catalpol-H had no significant change in the serum levels of TNF- $\alpha$, IL- $1 \beta$ and IL- 6 compared with the sham control group. Serum levels of TNF- $\alpha$, IL-1 $\beta$ and IL- 6 all significantly increased following BDL. Furthermore, compared with the BDL model group, catalpol-L and catalpol-H significantly reduced the levels of TNF- $\alpha$, IL- $1 \beta$ and IL- 6 , with catalpol-H producing a greater effect compared with catalpol-L.

Effects of catalpol on histological alterations of cholestatic mice. The histological alterations in liver tissues derived from cholestatic mice were evaluated by $\mathrm{H} \& \mathrm{E}$ staining. As shown in Fig. 2, treatment with catalpol-H alone had no marked effect on the histological morphology of the mouse liver tissues. In the BDL model group, inflammatory infiltration and hepatocyte damage in the liver tissues were observed, and subsequent treatment with catalpol markedly reduced hepatocyte necrosis and neutrophil infiltration, with catalpol-H producing a greater protective effect compared with catalpol-L.

Effects of catalpol on hepatocyte mitochondria and oxidative stress of cholestatic mice. Flow cytometry was performed to detect mitochondrial membrane potential of the extracted hepatocytes. The subsequent results of JC-1 distribution demonstrated that the mitochondrial membrane potential in the BDL model group was reduced compared with that in the sham group. In addition, catalpol-H had no notable effect on mitochondrial membrane potential in normal mouse liver cells; however, treatment with catalpol-L and catalpol-H significantly improved the mitochondrial membrane potential of liver cells in the cholestatic model mice (Fig. 3A). The ATP content of mitochondria was determined using a luciferase luciferin kit; the results revealed that ATP content was significantly lower in mitochondria in the BDL group compared with the sham control group, whereas treatment with catalpol significantly increased the content of ATP in mitochondria within the cholestatic mice compared with the BDL model group (Fig. 3B). The production of ROS in the mitochondria was measured by flow cytometry, and the results indicated that ROS generation was increased in the BDL model group 

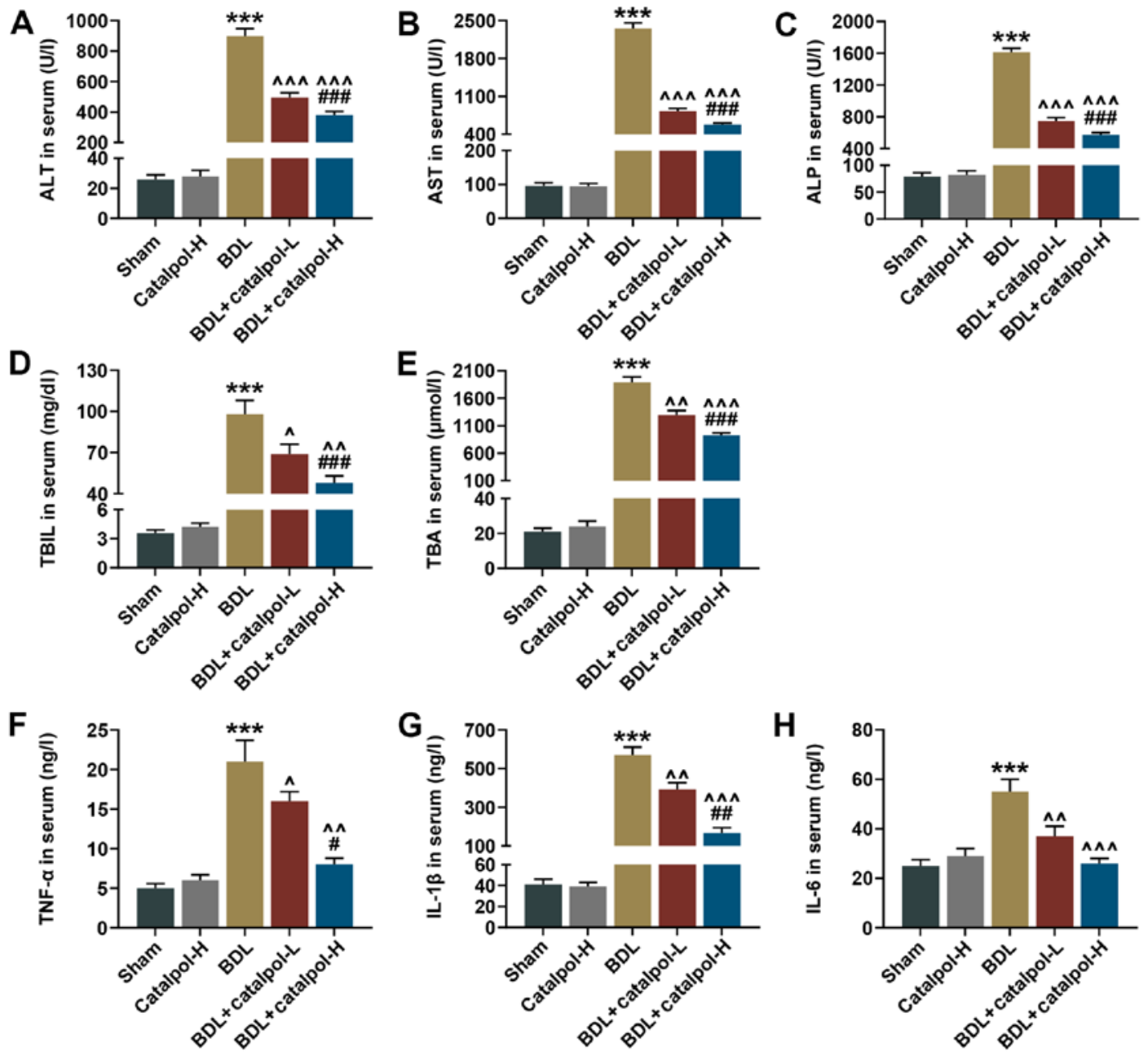

Figure 1. Effects of catalpol on serum biochemical indexes and inflammatory factor indexes in mice of different treatment groups. Liver function parameters of (A) ALT, (B) AST, (C) ALP, (D) TBIL and (E) TBA in mice in different treatment groups. Inflammatory factors, including (F) TNF- $\alpha$, (G) IL-1 $\beta$ and (H) IL-6, were assessed in the serum. ${ }^{* * *} \mathrm{P}<0.001$ vs. Sham; ${ }^{\#} \mathrm{P}<0.05,{ }^{\# \#} \mathrm{P}<0.01$ and ${ }^{\# \# \#} \mathrm{P}<0.001$ vs. Catalpol-H; ${ }^{\wedge} \mathrm{P}<0.05,{ }^{\wedge \wedge} \mathrm{P}<0.01$ and ${ }^{\wedge \wedge} \mathrm{P}<0.001$ vs. BDL. ALT, alanine aminotransferase; AST, aspartate aminotransferase; ALP, alkaline phosphatase; TBIL, total bilirubin; TBA, total bile acid; TNF- $\alpha$, tumor necrosis factor- $\alpha$; IL-1 $\beta$, interleukin-1 $\beta$; IL-6, interleukin-6; BDL, bile duct ligation; catalpol-H, catalpol-high treatment; catalpol-L, catalpol-low treatment.

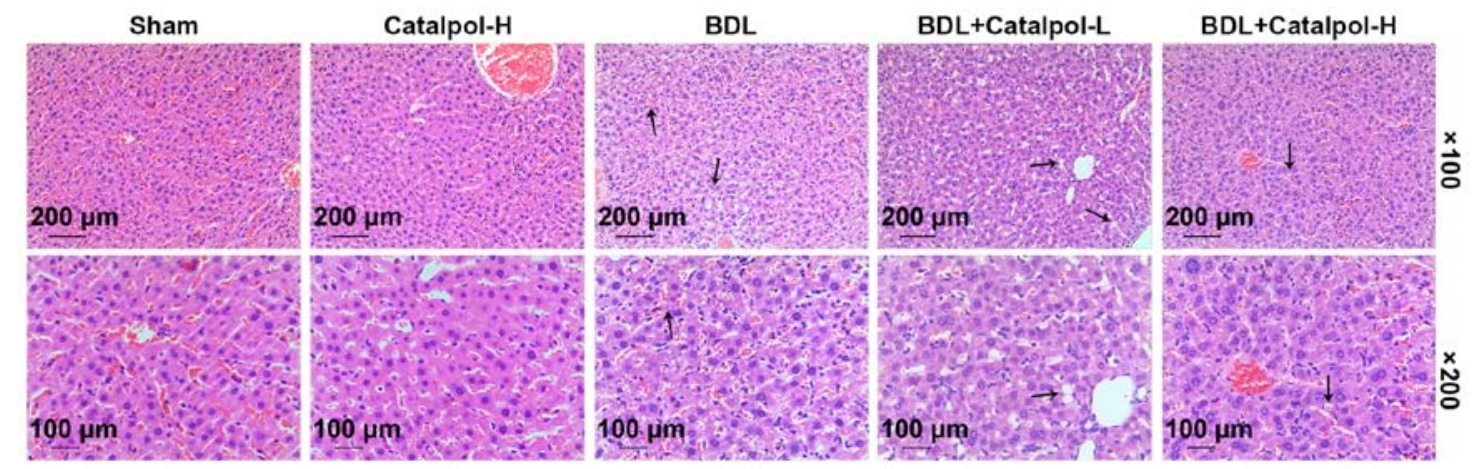

Figure 2. Effects of catalpol on histological alterations in mouse liver tissues. Mice underwent different treatments prior to hematoxylin and eosin staining of liver tissues. Catalpol-H, catalpol-high treatment; catalpol-L, catalpol-low treatment; BDL, bile duct ligation.

compared with the sham group, whereas catalpol significantly reduced the production of ROS compared with in the BDL model group, with catalpol-H producing a more significant effect compared with catalpol-L (Fig. 3C).

Intracellular GSH contents in the serum were also detected; as shown in Fig. 4A, GSH content was significantly reduced in the BDL model group compared with that in the sham control group, whereas treatment with catalpol significantly increased GSH content compared with the BDL model group. Once again, catalpol-H produced a greater protective effect compared with catalpol-L. Moreover, similar results were found with regards to GSH concentration in the 
A

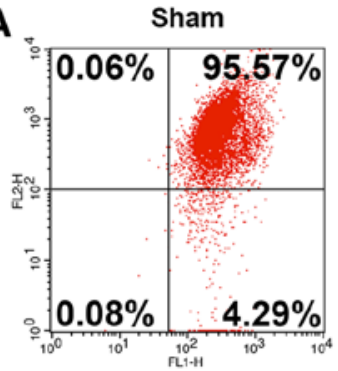

BDL+Catalpol-L

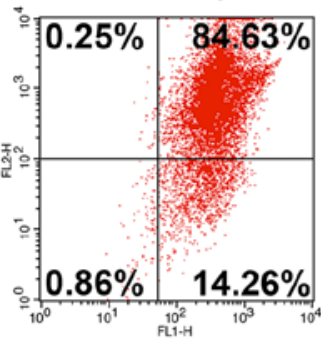

C

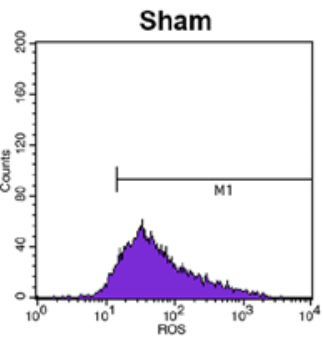

Catalpol-H

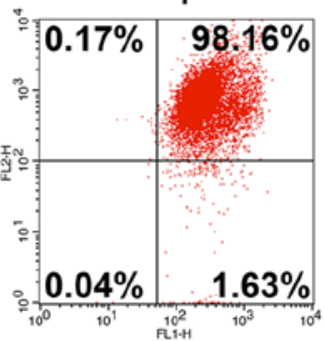

$\mathrm{BDL}+$ Catalpol-H
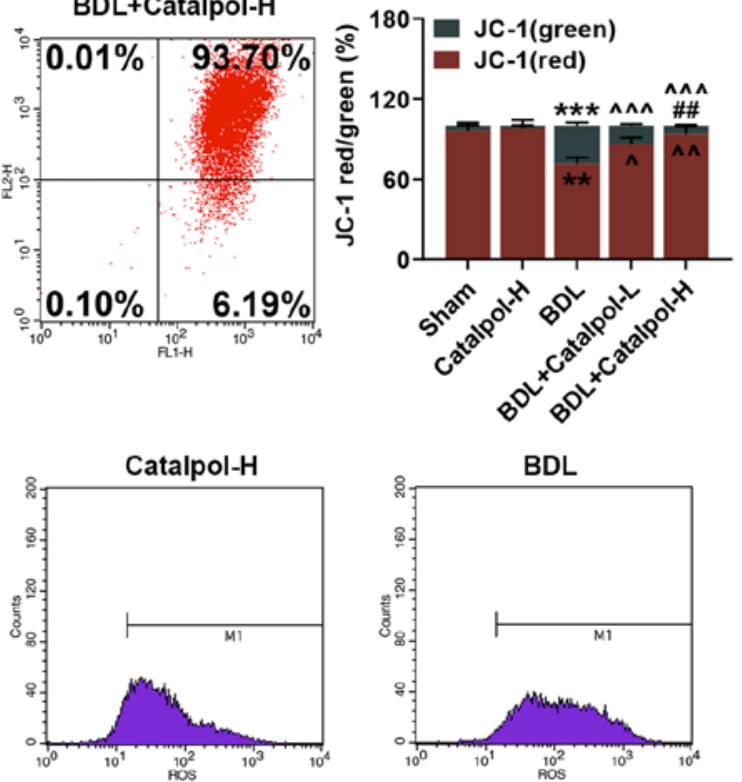

BDL

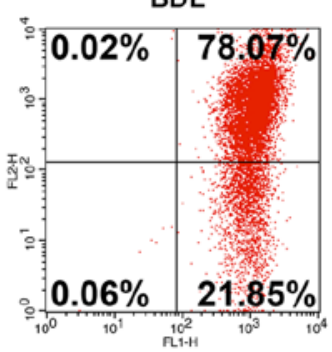

B

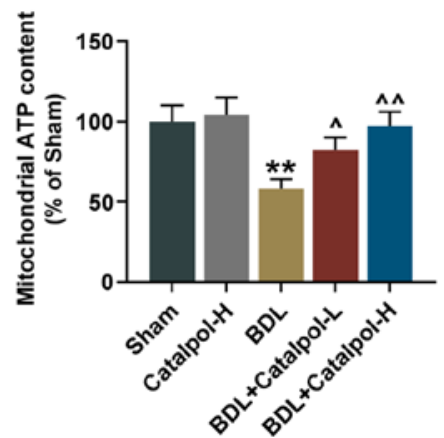

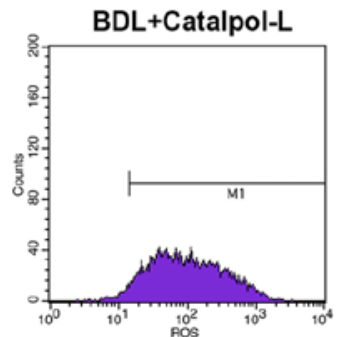
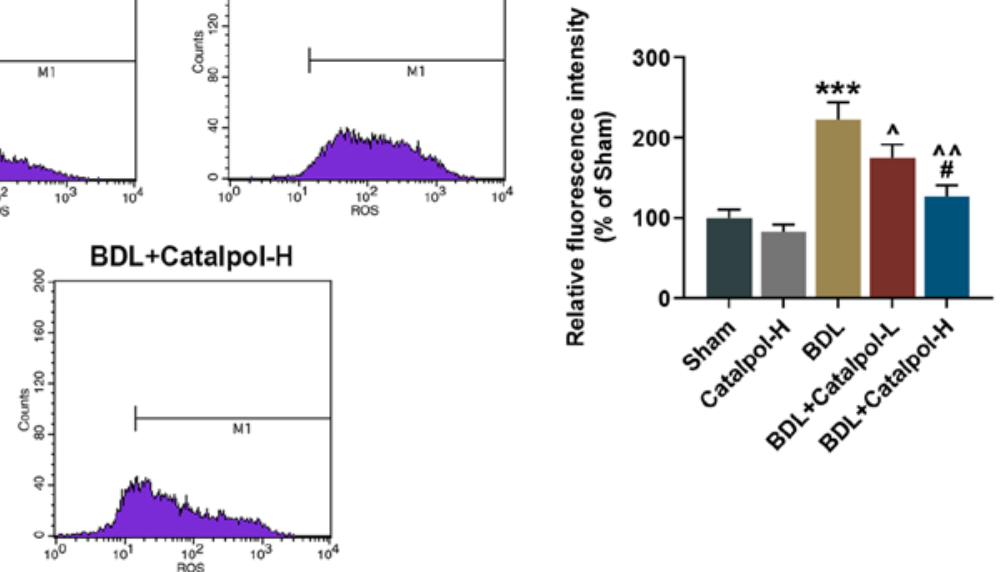

Figure 3. Effects of catalpol on hepatocellular mitochondria. (A) Flow cytometry detected JC-1 distribution, in order to measure the mitochondrial membrane potential in hepatocytes. (B) ATP content in hepatocellular mitochondria was determined. (C) ROS content in hepatocellular mitochondria was determined. ${ }^{* * *} \mathrm{P}<0.01$ and ${ }^{* * * *} \mathrm{P}<0.001$ vs. Sham; ${ }^{\#} \mathrm{P}<0.05$ and ${ }^{\# \#} \mathrm{P}<0.01$ vs. catalpol-H; ${ }^{\wedge} \mathrm{P}<0.05,{ }^{\wedge \wedge} \mathrm{P}<0.01$ and ${ }^{\wedge \wedge} \mathrm{P}<0.001$ vs. BDL. ROS, reactive oxygen species; catalpol-H, catalpol-high treatment; catalpol-L, catalpol-low treatment; BDL, bile duct ligation.

mitochondria (Fig. 4B). The contents of MDA in the serum and mitochondria were also assessed, and the data revealed that catalpol significantly reduced the total MDA content in the BDL cholestatic model mice (Fig. 4C and D), with catalpol-H producing a significantly more protective effect than catalpol-L.

Effects of catalpol on protein expression levels in the liver of cholestatic mice. As shown in Fig. 5, expression levels of proteins related to apoptosis and oxidative stress were measured. The results revealed that cytochrome $c$ protein expression was increased in the BDL model group, whereas cytochrome $c$ levels were significantly reduced by treatment with catalpol in the cholestatic model mice, with catalpol-H producing a greater protective effect compared with catalpol-L. With regards to the expression levels of proteins related to cell apoptosis, it was revealed that $\mathrm{Bcl}-2$ expression was significantly reduced, whereas Bax and cleaved caspase-3 were significantly increased in the BDL model group. Conversely, following treatment with catalpol, the protein expression levels were significantly altered and closer to the sham control sample levels. Once again, catalpol-H produced a more protective effect than catalpol-L. There were no notable changes to total caspase- 3 expression levels among the different control and treatment groups.

\section{Discussion}

Catalpol, which is an iridoid glycoside extracted from Rehmannia glutinosa, has an anti-ischemic effect (18-21). It has been suggested that catalpol could effectively protect promyelinating oligodendrocytes from apoptosis and myelination defects. Myelination defects may be induced by oxygen glucose deprivation, which catalpol can protect 

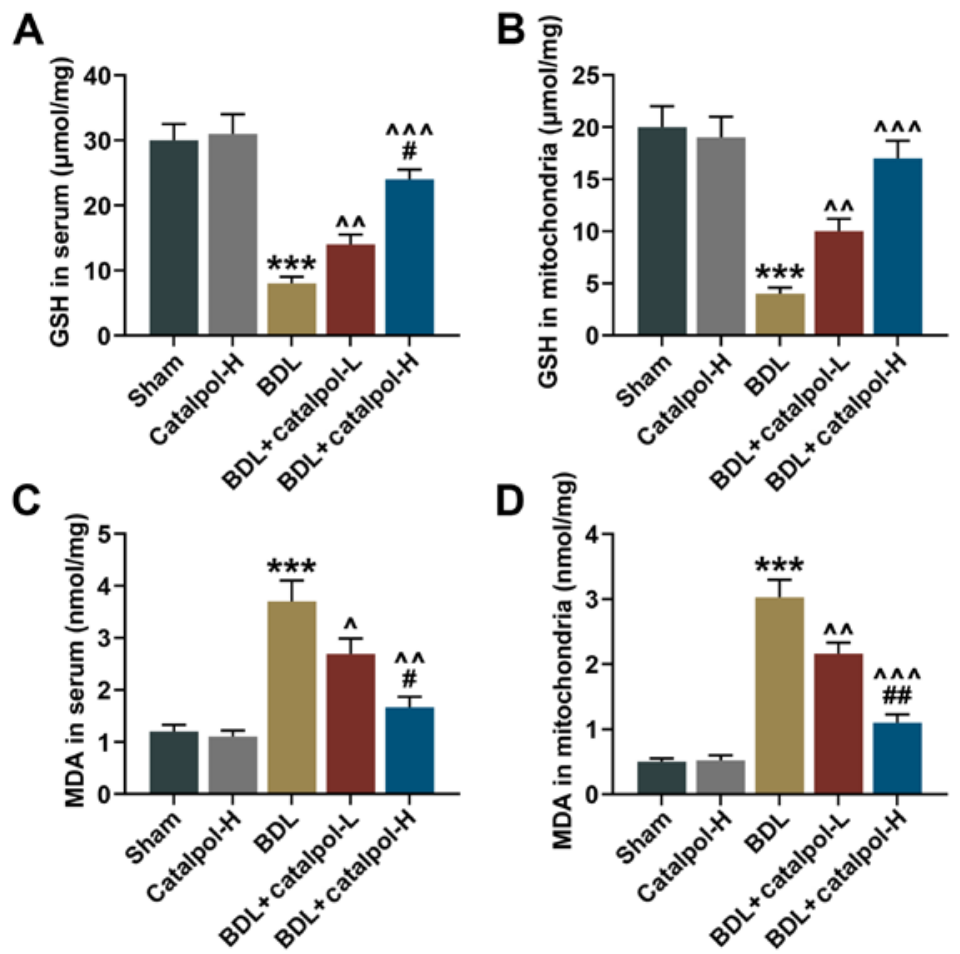

Figure 4. GSH and MDA in serum and mitochondria of liver cells of mice. GSH levels in (A) serum and (B) mitochondria of liver cells. MDA levels in (C) serum and (D) mitochondria of liver cells. ${ }^{* * * *} \mathrm{P}<0.001$ vs. Sham; ${ }^{\#} \mathrm{P}<0.05$ and ${ }^{\# \#} \mathrm{P}<0.01$ vs. catalpol- $\mathrm{H} ;{ }^{\wedge} \mathrm{P}<0.05,{ }^{\wedge} \mathrm{P}<0.01$ and ${ }^{\wedge \wedge} \mathrm{P}<0.001$ vs. BDL. GSH, glutathione; MDA, malondialdehyde; catalpol-H, catalpol-high treatment; catalpol-L, catalpol-low treatment; BDL, bile duct ligation.

against through suppressing $\mathrm{Ca}^{2+}$ overload. Catalpol has also been shown to inhibit mitochondrial damage and production of ROS (18). A recent study reported that catalpol had a protective role in diabetic nephropathy by regulating the synthesis of nitric oxide (22). The present study revealed that in a mouse model of cholestatic mice, catalpol exerted a protective function potentially via its antioxidant activities and regulation of mitochondrial membrane potential.

In this study, treatment with catalpol-H $(10 \mathrm{mg} / \mathrm{kg})$ had no significant effect on serum levels of ALT, AST, ALP, TBIL or TBA in mice in the sham control group, but it effectively reduced signs of liver damage caused by BDL in the cholestatic mouse model. ALT, AST, ALP, TBIL and TBA are all indicative of liver function (23). Recently, a study on the mechanism of andrographolide in the protection of cholestatic liver injury found that andrographolide exerted a certain protective effect on cholestatic liver injury, and the serum levels of the aforementioned indicators were decreased (24). The present results were consistent with this previous study, and preliminarily indicated that catalpol had a protective effect on liver injury caused by cholestasis.

Cholestasis, which is characterized by disordered bile formation and/or bile flow, is either an inherited or acquired disease, and can lead to the interruption of liver excretion of bile acids, enterohepatic circulation and may eventually develop into severe liver damage (25). Mitochondrial dysfunction is known to eventually induce cellular apoptosis and oxidative stress. Oxidative stress and mitochondrial damage are associated with the pathogenesis of liver cholestasis; oxidative stress can lead to cell death and organ damage, whereas the mitochondria are the main target of cytotoxic molecules, and mitochondrial damage can cause a cell energy crisis and release of cell death mediators (26). Excessive ROS is known to directly impair mitochondrial function, resulting in subsequent translocation of apoptosis-inducing factors from the mitochondria to the nuclei and eventually resulting in cell death (27). In addition, reduced mitochondrial membrane potential affects the opening of the mitochondrial permeability transition pore, which may cause mitochondria-dependent cell death (28). Studies have revealed that catalpol could scavenge ROS and enhance antioxidant abilities through improving mitochondrial functions that protect against oxidative attack (29-31). In the present study, the results demonstrated that catalpol could significantly increase mitochondrial membrane potential and reduce the levels of ROS in the hepatocytes of cholestatic mice. In addition, a previous study demonstrated that catalpol reduced oxidative stress in a colitis model, which in turn reversed the increase in MDA, and reductions in GSH and superoxide dismutase (SOD) in the colitis group (32). Cai et al (18) previously reported that catalpol ameliorated overproduction of ROS and protected pre-myelinating oligodendrocytes against ischemia-induced oxidative injury via the ERK1/2 signaling pathway.

GSH has a key role in the antioxidant defense system of cells and is also a key factor in maintaining the redox environment of cells. GSH protects cells from excessive ROS by reacting directly with free radicals in non-enzymatic reactions and acting as an electronic donor for GSH peroxidase to catalyze peroxide reduction (33). MDA is a marker of oxidative stress; a significant increase in MDA indicates a significant increase in lipid peroxidation (34). The results of the present study revealed that treatment with catalpol greatly increased mitochondrial ATP and GSH contents, and reduced the level of MDA, which suggested that catalpol may protect hepatocytes against oxidative stress through the induction of antioxidant generation 

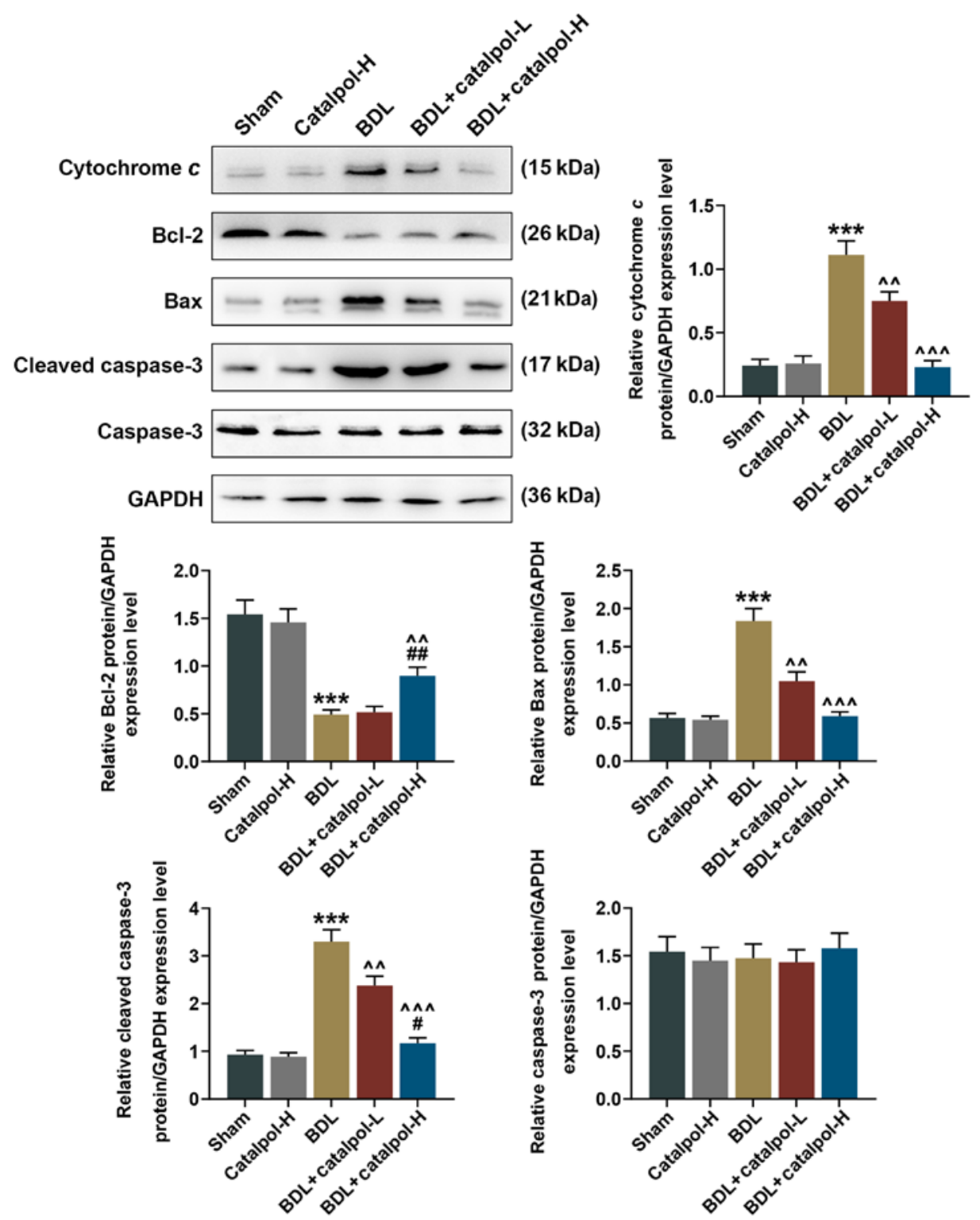

Figure 5. Expression levels of proteins related to apoptosis and oxidative stress (cytochrome c, Bcl-2, Bax, cleaved caspase-3 and caspase-3) in liver tissues, as measured by western blotting. ${ }^{* * * *} \mathrm{P}<0.001$ vs. Sham; ${ }^{~} \mathrm{P}<0.05,{ }^{\# \#} \mathrm{P}<0.01$ vs. catalpol-H; ${ }^{\wedge \wedge} \mathrm{P}<0.01,{ }^{\wedge \wedge} \mathrm{P}<0.001$ vs. BDL. catalpol-H, catalpol-high treatment; catalpol-L, catalpol-low treatment; BDL, bile duct ligation.

and a reduction in the generation of oxidizing products. These results indicated that the antioxidant pharmacological activity of catalpol may be produced through the enhancement of endogenous antioxidant enzymatic activities and through the inhibition of free radical generation (indicated by decreased levels of ROS). Catalpol was previously shown to increase the activity of GSH and reduce MDA levels, which thereby ameliorated cognitive deficits and attenuated oxidative damage in the brain of elderly mice induced by D-galactose (29). In addition, a previous study indicated that the protected effect of glycine on cholestasis may be related to its ability to protect the redox environment, prevent oxidative stress and maintain mitochondrial function (35). Consistently, the present study reported that following treatment with catalpol, the pathological changes of liver tissues in the BDL model mice could be alleviated. In addition, the functional indexes of liver mitochondria in cholestatic mice were evaluated, and the damage to the liver caused by cholestasis was found to result in the dissipation of the liver mitochondrial membrane potential, which was markedly reversed after the drug administration.

The current findings also revealed that catalpol greatly reduced the contents of pro-inflammatory factors in the serum derived from model mice in a concentration-dependent manner. A recent study indicated that IL- 6 and IL- $1 \beta$ may enhance the bile canaliculi dilatation caused by cholestatic antibiotics (36). It has also been found that ginsenoside can attenuate oxidative stress and the inflammatory response in rats with intrahepatic cholestasis (37). The present data demonstrated that the serum levels of AST, ALT, ALP and TBA, liver MDA, SOD activity and pathological damage of the cholestatic model mice were markedly reduced by catalpol. In addition, ginsenoside treatment was found to noticeably reduce the protein expression levels of TNF- $\alpha$, IL- 6 and IL-1 $\beta$ (37). These results are consistent with the present research results. In addition, the apoptotic and oxidative stress proteins, such as cytochrome $c, \mathrm{Bcl}-2$, Bax and caspase-3 were detected in the liver tissues, and a high concentration of 
catalpol significantly downregulated the expression of Bax, cytochrome $c$ and cleaved caspase 3, and upregulated Bcl-2 expression in the hepatocytes of cholestatic mice.

In conclusion, the current study revealed that catalpol protected the liver from cholestasis-mediated injury through its antioxidant effects, and its ability to improve mitochondrial membrane potential and regulate inflammatory cytokines. However, there are still some limitations in this study; for example, catalpol plays a protective role in the oxidative damage of cholestasis by targeting genes or regulating pathways, and the specific genes and pathways that catalpol interacts with need to be further studied. In addition, the findings need to be confirmed in clinical studies. Taken together, catalpol may act to protect hepatocytes from oxidative stress and could improve mitochondrial membrane potential, which is closely related to the recovery of cholestasis-mediated liver injury.

\section{Acknowledgements}

Not applicable.

\section{Funding}

No funding was received.

\section{Availability of data and materials}

The datasets used and/or analyzed during the current study are available from the corresponding author on reasonable request.

\section{Authors' contributions}

XG and JX made substantial contributions to study conception and design. Data acquisition, analysis and interpretation was conducted by XG, JX and HL, along with drafting and critically revising the manuscript. All authors agreed to be accountable for all aspects of the work in ensuring that questions related to the accuracy or integrity of the work are appropriately investigated and resolved. All authors read and approved the final version of the manuscript.

\section{Ethics approval and consent to participate}

This study was approved by the Ethics Review Board for Animal Studies of the Affiliated Yantai Yuhuangding Hospital of Qingdao University (approval no. SH20186242).

\section{Patient consent for publication}

Not applicable.

\section{Competing interests}

The authors declare that they have no competing interests.

\section{References}

1. Afonso MB, Rodrigues PM, Simao AL, Ofengeim D, Carvalho T, Amaral JD, Gaspar MM, Cortez-Pinto H, Castro RE, Yuan J, et al: Activation of necroptosis in human and experimental cholestasis. Cell Death Dis 7: e2390, 2016.
2. Vinken M: In vitro prediction of drug-induced cholestatic liver injury: A challenge for the toxicologist. Arch Toxicol 92: 1909-1912, 2018.

3. Squires JE and McKiernan P: Molecular mechanisms in pediatric cholestasis. Gastroenterol Clin North Am 47: 921-937, 2018.

4. Gomez-Ospina N, Potter CJ, Xiao R, Manickam K, Kim MS, Kim KH, Shneider BL, Picarsic JL, Jacobson TA, Zhang J, et al: Mutations in the nuclear bile acid receptor FXR cause progressive familial intrahepatic cholestasis. Nat Commun 7: 10713, 2016.

5. Ommati MM, Farshad O, Niknahad H, Arabnezhad MR, Azarpira N, Mohammadi HR, Haghnegahdar M, Mousavi K, Akrami S, Jamshidzadeh A, et al: Cholestasis-associated reproductive toxicity in male and female rats: The fundamental role of mitochondrial impairment and oxidative stress. Toxicol Lett 316 : 60-72, 2019.

6. Yin F, Yan J, Zhao Y, Guo KJ, Zhang ZL, Li AP, Meng CY and Guo L: Bone marrow mesenchymal stem cells repair $\mathrm{Cr}$ (VI)-injured kidney by regulating mitochondria-mediated apoptosis and mitophagy mediated via the MAPK signaling pathway. Ecotoxicol Environ Saf 176: 234-241, 2019.

7. Rajavel T, Packiyaraj P, Suryanarayanan V, Singh SK, Ruckmani K and Pandima Devi K: $\beta$-sitosterol targets Trx/Trx 1 reductase to induce apoptosis in A549 cells via ROS mediated mitochondrial dysregulation and p53 activation. Sci Rep 8: 2071, 2018.

8. Zhao X, Ren X, Zhu R, Luo Z and Ren B: Zinc oxide nanoparticles induce oxidative DNA damage and ROS-triggered mitochondria-mediated apoptosis in zebrafish embryos. Aquat Toxicol 180: 56-70, 2016.

9. Wang LL, Han L, Ma XL, Yu QL and Zhao SN: Effect of mitochondrial apoptotic activation through the mitochondrial membrane permeability transition pore on yak meat tenderness during postmortem aging. Food Chem 234: 323-331, 2017.

10. Xia Z, Wang F, Zhou S, Zhang R, Wang F, Huang JH, Wu E, Zhang $\mathrm{Y}$ and $\mathrm{Hu} \mathrm{Y}$ : Catalpol protects synaptic proteins from beta-amyloid induced neuron injury and improves cognitive functions in aged rats. Oncotarget 8: 69303-69315, 2017.

11. Huang JZ, Wu J, Xiang S, Sheng S, Jiang Y, Yang Z and Hua F: Catalpol preserves neural function and attenuates the pathology of Alzheimer's disease in mice. Mol Med Rep 13: 491-496, 2016.

12. Gao J, An L, Xu Y and Huang Y: Catalpol exerts an anti-epilepticus effect, possibly by regulating the Nrf2-Keap1-ARE signaling pathway. Med Sci Monit 24: 9436-9441, 2018.

13. Lin CM, Wang BW, Fang WJ, Pan CM, Shyu KG and Hou SW: Catalpol ameliorates neointimal hyperplasia in diabetic rats. Planta Med 85: 406-411, 2019.

14. Zhang H, Jia R, Wang F, Qiu G, Qiao P, Xu X and Wu D: Catalpol protects mice against Lipopolysaccharide/ D-galactosamine-induced acute liver injury through inhibiting inflammatory and oxidative response. Oncotarget 9: 3887-3894, 2018.

15. Zhang Y, Wang C, Jin Y, Yang Q, Meng Q, Liu Q, Dai Y, Cai L, Liu Z, Liu K and Sun H Activating the PGC- $1 \alpha /$ TERT pathway by catalpol ameliorates atherosclerosis via modulating ROS production, DNA damage, and telomere function: Implications on mitochondria and telomere link. Oxid Med Cell Longev 2018: 2876350, 2018

16. Park KS: Catalpol reduces the production of inflammatory mediators via PPAR-gamma activation in human intestinal Caco-2 cells. J Natural Med 70: 620-626, 2016.

17. National Research Council Committee for the Update of the Guide for the $\mathrm{C}$ and use of laboratory A: The National Academies Collection: Reports funded by National Institutes of Health. In: Guide for the care and use of laboratory Animals. National Academies Press (US) Copyright (C) 2011, National Academy of Sciences., Washington (DC), 2011.

18. Cai Q, Ma T, Li C, Tian Y and Li H: Catalpol protects Pre-myelinating oligodendrocytes against Ischemia-induced oxidative injury through ERK1/2 signaling pathway. Int J Biol Sci 12: 1415-1426, 2016

19. Xu D, Wang L, Jiang Z, Zhao G, Hassan HM, Sun L, Fan S, Zhou Z, Zhang L and Wang T: A new hypoglycemic mechanism of catalpol revealed by enhancing MyoD/MyoG-mediated myogenesis. Life Sci 209: 313-323, 2018.

20. Zeng YF, Wang R, Bian Y, Chen WS and Peng L: Catalpol attenuates IL-1 $\beta$ induced matrix catabolism, apoptosis and inflammation in rat chondrocytes and inhibits cartilage degeneration. Med Sci Monit 25: 6649-6659, 2019. 
21. Lin C, Lu Y, Yan X, Wu X, Kuai M, Sun X, Chen Q, Kong X, Liu Z, Tang Y, et al: Catalpol protects glucose-deprived rat embryonic cardiac cells by inducing mitophagy and modulating estrogen receptor. Biomed Pharmacother 89: 973-982, 2017.

22. Sun W, Gao Y, Ding Y, Cao Y, Chen J, Lv G, Lu J, Yu B, Peng M, Xu H, et al: Catalpol ameliorates advanced glycation end product-induced dysfunction of glomerular endothelial cells via regulating nitric oxide synthesis by inducible nitric oxide synthase and endothelial nitric oxide synthase. IUBMB Life 71: $1268-1283,2019$.

23. Ma X, Wen JX, Gao SJ, He X, Li PY, Yang YX, Wei SZ, Zhao YL and Xiao XH: Paeonia lactiflora Pall. regulates the NF- $\kappa$ B-NLRP3 inflammasome pathway to alleviate cholestasis in rats. J Pharm Pharmacol 70: 1675-1687, 2018.

24. Wang L, Cao F, Zhu LL, Liu P, Shang YR, Liu WH, Dong X, Bao HD, Gong P and Wang ZY: Andrographolide impairs alpha-naphthylisothiocyanate-induced cholestatic liver injury in vivo. J Natural Med 73: 388-396, 2019.

25. Wagner $M$ and Trauner M: Recent advances in understanding and managing cholestasis. F1000Research 5: F1000 Faculty Rev-705, 2016.

26. Heidari R and Niknahad H: The role and study of mitochondrial impairment and oxidative stress in cholestasis. Methods Mol Biol 1981: 117-132, 2019.

27. Baud O,Li J,Zhang Y,Neve RL, Volpe JJ and Rosenberg PA: Nitric oxide-induced cell death in developing oligodendrocytes is associated with mitochondrial dysfunction and apoptosis-inducing factor translocation. Eur J Neurosci 20: 1713-1726, 2004

28. Javadov S, Karmazyn M and Escobales N: Mitochondrial permeability transition pore opening as a promising therapeutic target in cardiac diseases. J Pharmacol Exp Ther 330: 670-678, 2009.

29. Zhang XL, Jiang B, Li ZB, Hao S and An LJ: Catalpol ameliorates cognition deficits and attenuates oxidative damage in the brain of senescent mice induced by D-galactose. Pharmacol Biochem Behav 88: 64-72, 2007.

30. Bi J, Wang XB, Chen L, Hao S, An LJ, Jiang B and Guo L: Catalpol protects mesencephalic neurons against MPTP induced neurotoxicity via attenuation of mitochondrial dysfunction and MAO-B activity. Toxicol In Vitro 22: 1883-1889, 2008.
31. Riddle A, Luo NL, Manese M, Beardsley DJ, Green L, Rorvik DA, Kelly KA, Barlow CH, Kelly JJ, Hohimer AR and Back SA: Spatial heterogeneity in oligodendrocyte lineage maturation and not cerebral blood flow predicts fetal ovine periventricular white matter injury. J Neurosc 26: 3045-3055, 2006.

32. Xiong Y, Shi L, Wang L, Zhou Z, Wang C, Lin Y, Luo D, Qiu J and Chen D: Activation of sirtuin 1 by catalpol-induced down-regulation of microRNA-132 attenuates endoplasmic reticulum stress in colitis. Pharmacol Res 123: 73-82, 2017.

33. Antus B: Oxidative stress markers in sputum. Oxid Med Cell Longev 2016: 2930434, 2016.

34. Shaker RA, Abboud SH, Assad HC and Hadi N: Enoxaparin attenuates doxorubicin induced cardiotoxicity in rats via interfering with oxidative stress, inflammation and apoptosis. BMC Oharmacol Toxicol 19: 3, 2018.

35. Heidari R, Ghanbarinejad V, Mohammadi H, Ahmadi A, Ommati MM, Abdoli N, Aghaei F, Esfandiari A, Azarpira N and Niknahad H: Mitochondria protection as a mechanism underlying the hepatoprotective effects of glycine in cholestatic mice. Biomed Pharmacother 97: 1086-1095, 2018.

36. Sharanek A, Burban A, Ciriaci N and Guillouzo A: Pro-inflammatory cytokines enhance dilatation of bile canaliculi caused by cholestatic antibiotics. Toxicol In Vitro 58: 51-59, 2019.

37. Xu YJ, Yu ZQ, Zhang CL, Li XP, Feng CY, Lei K, He WX and Liu D: Protective effects of ginsenosides on 17[Formula: See text]-Ethynyelstradiol-Induced intrahepatic cholestasis via anti-oxidative and anti-inflammatory mechanisms in rats. Am J Chin Med 45: 1613-1629, 2017. International (CC BY-NC-ND 4.0) License. 